\title{
La saisie émotive du kairos avec des enfants : entre acte philosophique et geste didactique
}

\section{Emotional grasping of the kairos in children talk: between philosophical act and didactical gesture}

\section{Claire Polo ${ }^{1}$, Kristine Lund $^{2}$}

$\underline{\text { Abstract: An essential gesture of animating a philosophical dialogue with children consists }}$ in grasping within their talk, an opportune word or turn of phrase, the kairos, and bouncing off it to advance reasoning. Based on the analysis of expert practices, we propose a typology of the emotional grasp of Kairos that reflects the tension between investigative and educational aims in these exchanges. Beyond the effect of surprise, regulation makes it possible to welcome and share one's emotions and to make them evolve into wonder, astonishment or doubt. Such trajectories are decisive for the future of the new idea. But other reactions are frequent, offering other opportunities for the current activity and children training in the long term.

Keywords: educational dialogue, emotional regulation, kairos, opportunity, philosophical inquiry.

Résumé: Un geste essentiel d'animation du dialogue philosophique avec des enfants consiste à saisir la parole opportune, le kairos, parmi leurs dires, et rebondir dessus pour faire avancer le raisonnement. Au cour de la tension entre visées d'enquête et éducative dans ces échanges, nous proposons ici, à partir de l'analyse de pratiques expertes, une typologie de la saisie émotive du kairos. Au-delà de l'effet de surprise, la régulation permettant d'accueillir, de partager ses affects, puis de les faire évoluer en émerveillement, étonnement ou doute est décisive pour le

${ }^{1}$ Claire POLO est Maîtresse de Conférences en Sciences de l'Education et de la Formation à l'université Lyon 2 (France), au sein du laboratoire Education, Cultures, Politiques. Elle développe une approche linguistique de

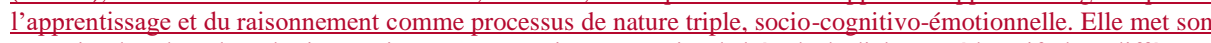
expertise dans l'analyse des interactions argumentatives au service de l'étude de dialogues éducatifs dans différents contextes. Elle a publié en 2020 Le Débat Fertile, explorer une controverse dans l'émotion. Claire1.polo@univlyon2.fr.

${ }^{2}$ Kristine Lund dirige le laboratoire d'excellence français Advanced Studies on Language Complexity. Elle est ingénieur de recherche CNRS au sein du laboratoire ICAR (Interactions, Corpus, Apprentissage, Représentations) à Ecole Normale Supérieure de Lyon. En s'intéressant aux diverses perspectives sur la collaboration et l'apprentissage pour les individus, les groupes et les communautés, elle développe un modèle qui permet de connecter des cadres épistémologiques différents. En 2020, elle a été élue Fellow de International Society of the Learning Sciences. Kristine.Lund@ens-lyon.fr. 
devenir de l'idée nouvelle. Mais d'autres réactions sont fréquentes, offrant d'autres opportunités pour l'activité en cours et la formation des enfants sur le long terme.

Mots-clefs: dialogue éducatif, enquête philosophique, kairos, opportunité, regulation émotionnelle. 


\section{La saisie émotive du kairos avec des enfants : entre acte philosophique et geste didactique}

\section{Introduction}

Les enfants constituent un public spécifique vis-à-vis de la philosophie, à la fois pour leur regard profane sans formation disciplinaire ni longue expérience du monde, et pour leur capacité de questionnement et leur ouverture d'esprit liée précisément à leur jeune âge. Ils sont visés depuis des décennies par des usages multiples de la philosophie appartenant au champ de la Philosophy for Children (P4C), qui ont en commun l'objectif de les amener à s'engager dans un véritable dialogue philosophique, et donc, comme pendant, le postulat de leur capacité à philosopher. Cependant, il existe un consensus sur la nécessité d'une intervention didactique pour, a minima, créer une situation qui les mette face à un problème philosophique, et les guider, plus ou moins activement et de façons différentes selon les écoles ${ }^{3}$, dans l'exploration proprement philosophique d'un tel problème. Ce travail d'animation de dialogue philosophique avec des enfants est généralement pris en charge par une personne adulte, avec ou sans formation disciplinaire, au statut d'enseignant ou de spécialiste de cette pratique, et requiert des gestes professionnels spécifiques. Parmi eux, savoir saisir la parole opportune, le kairos, parmi les dires enfantins et rebondir dessus pour faire avancer le raisonnement collectif, est essentiel. Cela dit, au-delà d'un geste professionnel, il s'agit d'un acte philosophique fondamental dont chaque personne doit pouvoir faire preuve, en dialogue philosophique, y compris donc, les enfants. Il y a là une tension entre situation didactique et adidactique ${ }^{4}$, se traduisant par la coexistence de différents contrats de communication, de telle sorte que jouer le jeu d'une exploration philosophique suppose parfois, pour les enfants, de sortir de leur rôle d'apprenant en transgressant le cadrage didactique proposé ${ }^{5}$. Pour la personne qui anime, la tension entre la visée d'enquête sur le problème posé et la visée d'éducation philosophique amène aussi à une posture ambivalente, qui peut parfois faire obstacle à la saisie du kairos. Nous proposons ici de nous intéresser tout particulièrement à l'émergence des émotions et leur régulation face au surgissement du kairos, en P4C, en nous questionnant sur leur rôle dans la pratique de la philosophie par le dialogue. Après la présentation d'un cadre théorique nécessairement interdisciplinaire pour appréhender un tel objet (1), nous précisons nos questions de recherches et notre méthode de travail à partir de l'étude de pratiques d'animation expertes (2), puis nous donnons à voir nos résultats, structurés sous la forme d'une typologie des saisies émotionnelles du kairos dans la dynamique interactionnelle de P4C (3). Enfin, une dernière section propose une discussion plus générale à partir de nos analyses, mettant en regard les opportunités offertes

\footnotetext{
${ }^{3}$ On note notamment en France des pratiques d'inspiration lipmanienne venues d'Outre-Atlantique, et d'autres développées en France sous l'impulsion de Michel Tozzi.

${ }^{4}$ A. Kohler, «Cheminement de pensée des élèves lors de leur schématisation du destin : une analyse de logique naturelle », in A. Fournel \& J.-P. Simon (Coord.), Enfants et adolescents en discussion philosophique. UGA Éditions, à paraître.

${ }^{5}$ C. Polo, "Jouer le jeu et enfreindre les règles : stratégies d'orientation discursive et argumentation dans une démonstration d'atelier philosophique", in J.-P. Simon \& M. Tozzi (Eds.), Paroles de philosophes en herbe, Editions de l'Université de Grenoble, 2017, p. 99-118.
} 
par chacun de ces types de saisie émotionnelle du kairos et leurs effets potentiels pour la recherche philosophique en train de se faire et pour la formation des enfants sur le long terme (4).

\section{Cadre contextuel et conceptuel : saisir le kairos et s'émouvoir pour penser ensemble}

Pour comprendre la dimension émotionnelle de la saisie du kairos en dans le dialogue philosophique, il nous paraît nécessaire de puiser des ancrages conceptuels à plusieurs traditions de recherche. Après avoir parcouru la littérature sur le kairos en $\mathrm{P} 4 \mathrm{C}$ (1.1), nous revenons sur le rôle des émotions dans tout raisonnement collectif et les enjeux de régulation émotionnelle associés (1.2), ce qui permet de préciser notre conception du dialogue philosophique comme une activité socio-cognitivo-émotionnelle (1.3).

\subsection{Le kairos au cœur de la tension enquête-éducation en P4C}

La pratique de la $\mathrm{P} 4 \mathrm{C}$ est traversée par la tension entre une visée d'enquête et une visée éducative. La première repose sur le postulat selon lequel les enfants constituent des «interlocuteurs valables $»^{6}$, capables de penser au même titre que les adultes. Dès lors, l'ensemble des personnes présentes est conçu comme engagé dans une activité commune (Polo, 2017, Kohler, à paraître) de dialogue visant à explorer philosophiquement des problèmes partagés au moins le temps d'une séance ${ }^{7}$. La pensée en acte dans un tel dialogue s'exprimerait à la fois avec spontanéité et sincérité, chaque personne étant authentiquement occupée à chercher à mieux comprendre la ou les question(s) abordée(s). Le principe de ce que Lipman ${ }^{8}$ appelle la Communauté de Recherche Philosophique (CRP) résiderait alors dans le pari selon lequel l'enquête collective permet d'ouvrir des horizons qui dépasseraient la somme de ce que chaque individu peut imaginer pour appréhender le(s) concept(s) à l'étude. Un tel processus d'exploration à travers le dialogue suppose que les raisonnements individuels soient rendus les plus transparents possibles, afin que leur richesse soit visible et appropriable par autrui ${ }^{9}$. Ainsi, il devient possible de conceptualiser en s'appuyant sur les dires des autres ${ }^{10}$, et même de faire émerger des synthèses traduisant l'état d'avancement de l'enquête ${ }^{11}()$. Dans cette perspective,

\footnotetext{
${ }^{6}$ J. Lévine, «La notion de «monde philosophique des enfants » : utopie ou nécessité ? Présupposés, place, limites ?”, in M. Tozzi, Apprendre à philosopher par la discussion, De Boeck Supérieur, 2007, p. 93-107.

${ }^{7}$ A. Fournel \& A.-N. Perret-Clermont, "Quel est le problème du problème ? Une analyse du problème supposé commun dans un atelier philosophique avec des collégiens", in A. Fournel \& J.-P. Simon (Coord.), Enfants et adolescents en discussion philosophique., UGA éditions, à paraître.

${ }^{8}$ M. Lipman, Thinking in Education (2e éd.). Cambridge University Press, 2003 ; M. Lipman, À l'école de la pensée. Enseigner une pensée holistique, De Boeck Supérieur, 2006.

${ }^{9}$ C. Polo, «Les gestes métaphoriques comme construction collective d'analogies cognitives », in A. Fournel, J.-P. Simon, S. Lagrange-Lanaspre, J.-M. Colletta (Coord.), Philosopher avec les enfants, Presses universitaires BlaisePascal, 2020, p. 461-484

${ }^{10} \mathrm{P}$. Roiné, « Reprises et représentations du discours de l'élève dans le discours de l'enseignant : un"triple jeu discursif" pendant les Discussions à Visée Philosophique », in A. Fournel \& J.-P. Simon (Coord.), Enfants et adolescents en discussion philosophique. UGA Éditions, à paraître.

11 Voir C. Polo, S. Lagrange-Lanaspre, "Metaphorical Reasoning Together: Embodied Conceptualization in a Community of Philosophical Inquiry", in K. Lund, G. Niccolai, E. Lavoué, C. Hmelo-Silver, G. Gweon, M. Baker (Eds), A Wide Lens: Combining Embodied, Enactive, Extended, and Embedded Learning in Collaborative Settings: 13th International Conference on CSCL, (1), 2019, p. 424-431. Ainsi que L. Lebas-Fraczak, « Les enfants
} 
l'animation de CRP consisterait principalement à susciter et suivre les cheminements de pensée des enfants, en les aidant à les expliciter afin qu'ils soient mis en commun au service de l'approfondissement de la discussion. La situation est alors conçue comme adidactique ${ }^{12}:$ c'est $^{\text {' }}$ une pratique philosophique dans laquelle une personne a un rôle particulier de facilitation des échanges, comme cela peut être le cas dans une CRP entre adultes, mais sans statut de sachant devant enseigner son savoir. L'orientation éducative est ici minimale : les enfants apprennent à philosopher en philosophant. Le contrat de communication qui régit les interactions se veut à ce titre horizontal et collaboratif ${ }^{13}$.

Cependant, la $\mathrm{P} 4 \mathrm{C}$ admet également une visée éducative très claire, qui puise sa source dans la conviction de ce que Tozzi ${ }^{14}$ appelle la «philosofiabilité » enfantine, l'éducabilité philosophique des enfants. De ce point de vue, les questions abordées constituent des problèmes-prétextes servant à développer des habiletés de pensée à long terme. Dans cette appréhension de la situation comme didactique ${ }^{15}$, le travail d'animation est crucial pour guider les enfants vers l'appropriation de cognitives et métacognitives ${ }^{16}$. Au niveau des compétences cognitives, Lipman cible l'apprentissage du triptyque des pensées créative, critique et attentive ou bienveillante (caring) ${ }^{17}$ via l'acquisition d'habiletés de penser ${ }^{18}$. Tozzi, lui, distingue problématisation, conceptualisation et argumentation ${ }^{19}$. Enfin, Baker ${ }^{20}$ pointe également les compétences cognitives associées à l'apprentissage du dialogue lui-même. Au niveau métacognitif, Pierrisnard ${ }^{21}$ a mis en évidence l'importance de la spiralité dans la démarche de Discussion à Visée Philosophique (DVP) avec des enfants, qui les invite à un retour sur la discussion au fil des synthèses successives comme autant de boucles réflexives. En DVP, soit la pratique de la $\mathrm{P} 4 \mathrm{C}$ selon la «méthode Tozzi », le dispositif de discussion lui-même favorise cette réflexivité par le fait de confier des rôles précis aux enfants (attribution de la parole, reformulation, prise de notes, participation au dialogue philosophique, etc.), de les échanger en milieu de séance, et de dédier un temps final à l'évaluation de la façon dont ces missions ont été assurées, à la fois par l'auto-évaluation des personnes concernées, et le retour de celles et ceux qui avaient un rôle d'observation de l'activité des autres. Cette visée éducative explique que des éléments du script interactionnel effectif des dialogues relevant de la P4C présentent

font-ils spontanément des synthèses dans les discussions à visée philosophique ?", in A. Fournel \& J.-P. Simon (Coord.), Enfants et adolescents en discussion philosophique. UGA Éditions, à paraître.

${ }_{12} \mathrm{~A}$. Kohler, "Cheminement de pensée des élèves lors de leur schématisation du destin : une analyse de logique naturelle », in A. Fournel \& J.-P. Simon (Coord.), Enfants et adolescents en discussion philosophique. UGA

Éditions, à paraitre.

${ }^{13} \mathrm{C}$. Polo, «Jouer le jeu et enfreindre les règles : stratégies d'orientation discursive et argumentation dans une démonstration d'atelier philosophique », in J.-P. Simon \& M. Tozzi (Eds.), Paroles de philosophes en herbe, Editions de l'Université de Grenoble, 2017, p. 99-118.

${ }^{14}$ M. Tozzi, «Le kairos dans une DVDP : une éthique de l'intervention pour l'émergence de sujets pensants », Diotime (en ligne) 60/2014 (4).

${ }^{15}$ A. Kohler, « Cheminement de pensée des élèves lors de leur schématisation du destin : une analyse de logique naturelle», art. cit.

${ }^{16}$ C. Pierrisnard, «L'entretien de co-explicitation au service de la recherche collaborative », Phronesis 6 (1) /2017, p. $153-165$.

${ }_{17}$ M. Lipman, Thinking in Education, op. cit.

${ }^{18}$ M. Lipman, À l'école de la pensée, op. cit.

${ }^{19}$ M. Tozzi, «Une approche par compétences en philosophie ? », Rue Descartes, 73(1)/2012, p. 22-51.

${ }^{20}$ M. Baker, «Les pédagogies dialogiques : raisonnement, conceptualisation et argumentation », in A. Fournel \&

J.-P. Simon (Coord.), Enfants et adolescents en discussion philosophique, UGA éditions, à paraître.

${ }^{21}$ C. Pierrisnard, «L'entretien de co-explicitation au service de la recherche collaborative », art. cit.

Mis en forme : Couleur de police : Texte 1

Mis en forme : Couleur de police : Texte 1 
des similitudes avec les aspects communicationnels classiques du contrat didactique en milieu scolaire, d'autant que l'animation est souvent assurée par des personnes ayant habituellement des fonctions enseignantes ${ }^{22}$. En particulier, la personne en situation d'animation se retrouve fréquemment dans une posture de sélection, d'évaluation et d'éventuelle institutionnalisation des propositions faites par les enfants.

Nous tenons à préciser que, si nous avons souligné le terme de CRP pour décrire la visée d'enquête de la $\mathrm{P} 4 \mathrm{C}$, et décrit des éléments de la méthode Tozzi pour témoigner de sa visée éducative, cela ne sous-entend pas que ces courants s'opposent sur ces plans. En effet, si une multitude de pratiques différentes existent dans le champ de la $\mathrm{P} 4 \mathrm{C}$, et s'il y a bien des distinctions entre les approches lipmanienne et tozzienne, toutes sont traversées par cette tension entre visée d'enquête et visée éducative. C'est sans doute plutôt dans la façon de chercher à tenir ensemble ces deux dimensions que ces écoles diffèrent : quand et comment l'une de ces visées est à privilégier par rapport à l'autre, dans la conception de dispositifs et leur animation concrètes.

En particulier, ces préférences pour une méthode ou une autre s'incarnent dans la façon dont on cherche à tirer le fil des pensées en émergence dans le dialogue, par des microdécisions d'animation, qui laissent plus ou moins s'ouvrir ou, au contraire, referment ce que Kohler (à paraître) nomme les «cheminements de pensée » initiés par les enfants. En effet, cette tension structurante entre enquête et éducation rend l'animation de CRP ou DVP ambivalente au plan de ses pratiques. Ainsi, une séance avec un objectif principal, par exemple, explorer le thème de la justice, peut se retrouver subdivisée en tâches aux consignes qui peuvent parfois entrer en contradiction : donner des exemples pour définir l'injustice / réfléchir à ce qui peut motiver à dire «c'est pas juste »- ce qui recouvre alors potentiellement un champ plus large que la question de la définition de la justice, comme la formulation d'un acte de langage visant à exprimer un désaccord (Polo, 2017). Par ailleurs, le souci de prendre en charge un rythme de discussion propice au maintien de l'attention et à l'avancement de la pensée ${ }^{23}$ peut conduire à des interventions d'animation multiples qui ne laissent pas toujours le temps et la place aux enfants de déployer pleinement leurs raisonnements. Les pratiques d'animation tendent souvent à orienter la discussion vers la reconstruction de définitions philosophiques classiques préexistantes, se rapprochant ici du cours dialogué ${ }^{24}$. Enfin, il arrive fréquemment, en contradiction avec la perception de l'animation comme l'explicitation et le suivi des cheminements de pensée des enfants, que des idées valables ne soient pas reprises voire même perçues par l'animateur ou l'animatrice-). Cette ambivalence dans les pratiques ${ }^{25}$ d'animation

${ }^{22} \mathrm{C}$. Polo, «Jouer le jeu et enfreindre les règles : stratégies d'orientation discursive et argumentation dans une démonstration d'atelier philosophique », in J.-P. Simon \& M. Tozzi (Eds.), Paroles de philosophes en herbe, Editions de l'Université de Grenoble, 2017, p. 99-118.

${ }^{23}$ C. Pierrisnard, «L'entretien de co-explicitation au service de la recherche collaborative », art. cit.

${ }^{24}$ Voir Y. Kebir, V. Boutet, A. Specogna, V. Saint Nizier de Almeida, « Le guidage en DVP : apport de l'analyse interlocutoire et des verbalisations en auto-confrontation », in A. Fournel \& J.-P. Simon (Coord.), Enfants et adolescents en discussion philosophique, UGA Éditions, à paraître ; et A. Kohler, "Cheminement de pensée des élèves lors de leur schématisation du destin : une analyse de logique naturelle », art. cit.

${ }^{25}$ Voir C. Polo, «Les gestes métaphoriques comme construction collective d'analogies cognitives », in A. Fournel, J.-P. Simon, S. Lagrange-Lanaspre, J.-M. Colletta (Coord.), Philosopher avec les enfants, Presses universitaires Blaise-Pascal, 2020, p. 461-484 ; C. Polo, « Mise en scène argumentative des sujets et régulation émotionnelle en CRP », in A. Fournel \& J.-P. Simon (Coord.), Enfants et adolescents en discussion philosophique. UGA éditions, à paraître. Et aussi : V. Delille, N. Markevitch Frieden \& G. Jeanmart, « La pensée à l'œuvre dans une discussion 
ne saurait être pleinement expliquée par la difficulté de cette tâche et notamment par l'arbitrage sur le vif entre les différents buts assignés à l'activité, car elle se retrouve également dans les attentes formulées à l'égard de la $\mathrm{P} 4 \mathrm{C}$ par les personnes qui animent de telles séances. En effet, des praticiens et praticiennes affirment effectivement cibler l'acquisition par les enfants de concepts disciplinaires ${ }^{26}$; et tous et toutes espèrent au moins «que de la philosophie se produise ». L'avènement du kairos est au cœur des attentes projetées sur l'animation de séances de $\mathrm{P} 4 \mathrm{C}$, dont cette première définition incarne très bien l'ambivalence :

l'animateur ne veut rien "faire dire", même s'il aimerait que "ça sorte" : dans les faits, "ça vient" (ou pas), et pas forcément quand il le souhaiterait [...] Par exemple s'il "attend" une définition (tout en étant prêt à en faire le deuil), la balle est dans le camp du discutant ou du groupe, qui peut botter en touche... Mais il peut "sauter" sur le kairos de tout embryon de définition pour relancer, approfondir : c'est alors le discutant, qui en choisissant de s'inscrire dans cet espace, lui donne forme ${ }^{27}$.

En effet, à l'intersection entre visées d'enquête et éducative, le kairos est par excellence ce qui est recherché en $\mathrm{P} 4 \mathrm{C}$, et ce dont l'animation doit savoir se saisir, alors même qu'il est par définition éclair furtif dont on ne peut maîtriser l'avènement. Emergeant dans la dynamique interactionnelle, il doit son nom à la métaphore du dieu grec de l'instant, imprévisible ${ }^{28}$, et désigne à la fois un moment opportun à ne pas rater ${ }^{29}$ et une pépite susceptible de faire advenir la philosophie. En tant qu'opportunité, toute appréhension du kairos est repose sur des critères subjectifs : il dépend de l'évaluation d'une parole comme méritant d'être saisie, sur l'instant, et de la perception de la bonne façon de rebondir dessus pour favoriser sa pleine éclosion. Or ces jugements subjectifs sont étroitement liés aux objectifs alors considérés comme prioritaires : approfondir l'enquête, reconstruire une définition classique, souligner une habileté de penser à l'œuvre, faire circuler la parole, etc. Cela explique qu'il n'existe pas de définition unique et stable du kairos en P4C. Deux acceptions différentes semblent cependant pouvoir être opposées, l'une extensive, où presque tout peut être considéré comme tel, et l'autre, restrictive, où il demeure une perle rare. L'approche 'tout-kairos' a le mérite de pointer que celui-ci peut survenir sous des formes extrêmement variées, et ne saurait être réduit à un type de contribution, puisqu'il peut s'agit aussi bien d'une question pertinente, d'un questionnement de la question, d'une demande de définition, d'un embryon de définition par un exemple, d'un synonyme, d'un attribut du concept, d'une réponse à la question posée, d'un argument la justifiant ou s'y opposant ${ }^{30}$. L'approche restrictive du kairos, elle, met en avant sa nouveauté, qui la distingue

philosophique : l'écouter, l'entendre, l'exploiter. Former à l'animation par l'observation minutieuse d'une discussion philosophique filmée », in J.-P. Simon \& M. Tozzi (Coord.), Paroles de philosophes en herbe. Editions de l'Université de Grenoble, 2017, p. 239-260.

${ }^{26}$ Y. Kebir et al., «Le guidage en DVP : apport de l'analyse interlocutoire et des verbalisations en autoconfrontation », art. cit.

${ }^{27}$ M. Tozzi, «Le kairos dans une DVDP : une éthique de l'intervention pour l'émergence de sujets pensants », Diotime (en ligne) 60/2014 (4).

${ }^{28}$ Ibid.

${ }^{29}$ C. Pierrisnard, «L'entretien de co-explicitation au service de la recherche collaborative », Phronesis 6 (1) /2017, p. 153-165.

${ }^{30}$ M. Tozzi, «Le kairos dans une DVDP : une éthique de l'intervention pour l'émergence de sujets pensants », art. cit. 
des « opportunités classiques $»^{31}$ par sa capacité à surprendre la personne qui anime (et qui a peut-être déjà exploré de nombreuses fois la thématique, y compris même par une formation disciplinaire) :

[Q]uelque chose de surprenant surgit dans l'intervention d'un participant, quelque chose qui prend au dépourvu l'animateur et parfois le groupe, quelque chose d'un peu miraculeux qui s'invite, comme une grâce ou un cadeau. Il peut s'agir d'un lien inédit (lien entre la réflexion en cours et la réalité ou connexion nouvelle entre idées); d'une question vive qui atteste une pensée en recherche; d'un pas de côté qui opère un renversement de la perspective (par exemple on dézoome pour voir plus large, ou on ouvre une percée) ; d'une lecture nouvelle du problème sur lequel le groupe travaille depuis un moment ; ou bien d'une nouvelle structuration qui vient cristalliser le processus de réflexion commune. De telles interventions peuvent provoquer un vif étonnement et agir comme un révélateur, ou décontenancer et ne pas être perçues dans leur puissance novatrice ${ }^{32}$.

Tozzi $^{33}$ reconnait lui aussi la distinction entre kairos « relativement prévisible » et «surprise complètement inédite », supposant une capacité d'écoute cognitive «plus subtil[e]».

Reste qu'aussi bien l'approche extensive que restrictive du kairos s'accordent sur le fait que sa saisie est un geste professionnel d'animation qui peut être difficile pour les novices mais qui s'apprend ${ }^{34}$, et s'affûte avec l'expérience ${ }^{35}$. Savoir saisir le kairos, c'est être capable de s'ajuster à l'imprévu ${ }^{36}$, ce qui passe, en $\mathrm{P} 4 \mathrm{C}$, par la suspension de tout objectif lié à l'atteinte d'un contenu spécifique ${ }^{37}$. C'est, bien sûr, en temps réel, la fameuse pratique du « rebond $»^{38}$, qui permet à la personne en situation d'animation d'exploiter un kairos enfantin pour l'avancement de l'enquête. Mais une pratique d'animation vertueuse doit aussi susciter de telles pépites, par l'établissement d'un climat «kairogène », et disposer d'un état d'écoute et de concentration propice à la reconnaissance du kairos lorsqu'il survient, ce qui suppose un travail au-delà de l'instant, sur l'ensemble de la séance, voire de la vie. Un tel climat, selon Tozzi ${ }^{39}$, repose sur l'établissement et le maintien de quelques règles fondamentales garantissant à chaque personne

31 Toutefois, Dellile et ses collègues reconnaissent l'intérêt, en animation, de « reconnaître et souligner ces moments où la pensée propre d'un individu ou d'un groupe se réapproprie une grande question faisant partie du bagage culturel humain », même si cela est plus prévisible, et n'entre pas, pour elles, dans la définition du kairos. ${ }^{32}$ V. Delille et al., « La pensée à l'œuvre dans une discussion philosophique : l'écouter, l'entendre, l'exploiter. Former à l'animation par l'observation minutieuse d'une discussion philosophique filmée », art. cit., p. 241-242.

${ }^{33}$ M. Tozzi, « Le kairos dans une DVDP : une éthique de l'intervention pour l'émergence de sujets pensants », art. cit.

${ }^{34}$ Voir V. Delille et al., « La pensée à l'œuvre dans une discussion philosophique : l'écouter, l'entendre, l'exploiter. Former à l'animation par l'observation minutieuse d'une discussion philosophique filmée », art. cit. ; C. Pierrisnard, «L'entretien de co-explicitation au service de la recherche collaborative », art. cit. ; M. Tozzi, "Une approche par compétences en philosophie ?", art. cit.

${ }^{35}$ Nous entendons ici « geste professionnel » au sens de F. Pana-Martin : «Les gestes professionnels englobent les gestes du métier pour les (re)situer, les (re)lire, les (re)lier à l'activité professionnelle en jeu, en lui donnant un cadre plus personnel. [...] Il s'agit de l'intuition de l'instant qui montre que le formateur est présent et à l'écoute. ». (Les gestes professionnels des formateurs d'enseignants en situation d'accompagnement individualisé. Thèse de sociologie, CNAM,2015, p. 43).

${ }^{36}$ A. Jorro, Professionnaliser le métier d'enseignant, ESF, 2002.

${ }^{37}$ M. Tozzi, «Une approche par compétences en philosophie ? », art. cit.

${ }^{38} \mathrm{M}$. Tozzi, «Le kairos dans une DVDP : une éthique de l'intervention pour l'émergence de sujets pensants », art. cit.

${ }^{39}$ M. Tozzi, « Le kairos dans une DVDP : une éthique de l'intervention pour l'émergence de sujets pensants », art. cit. 
la liberté de penser, l'absence de jugement disqualifiant à son endroit, le droit de se taire, et un sentiment de confiance et de sécurité.

Cependant, saisir le kairos est aussi, si on appréhende la situation sous un angle adidactique, un acte philosophique essentiel qu'il revient à toutes les personnes présentes de pratiquer. En effet, l'animation ne saurait, seule, créer et entretenir un climat kairogène, dont la responsabilité échoit également au groupe. L'enfant doit en effet se montrer capable de produire un kairos, soit non seulement de laisser émerger en lui ou en elle un embryon de pensée nouvelle, mais également d'être «élève du kairos », autonome dans la gestion des aspects temporels de son intervention ${ }^{40}$, c'est-à-dire de le communiquer au bon moment et de la bonne façon. De plus, en tant que philosophe, l'enfant doit aussi développer une disposition d'écoute lui permettant de saisir, à son niveau, le kairos émergeant dans la parole d'autrui.

\subsection{Raisonnement collectif et régulation émotionnelle}

Pour mieux comprendre la saisie du kairos en $\mathrm{P} 4 \mathrm{C}$, revenons sur la dimension affective de tout raisonnement, a fortiori collectif et à propos d'une question ouverte telle que les problèmes philosophiques. Les émotions, dans l'argumentation en groupe, jouent à la fois des fonctions sociales et cognitives fondamentales ${ }^{41}$. Au plan social, la manifestation des ressentis quant à la préservation de leur face ${ }^{42}$ des différentes personnes participant à une discussion, conditionne l'alignement de leur posture de façon à s'engager, en tant que groupe, dans un véritable discours d'exploration ${ }^{43}$, processus indispensable à l'enquête. Au plan cognitif, le cadrage émotionnel du problème par combinaison de marques linguistiques explicites et faisceaux d'indices plus implicites oriente le discours vers la défense de thèses argumentatives spécifiques ${ }^{44}$.

Favoriser un fonctionnement des émotions en groupe susceptible de faire avancer la pensée peut être compris comme relevant de la régulation émotionnelle de l'activité. Dans le champ éducatif, cette notion est généralement utilisée dans une connotation réactive, c'est-à-dire comme réaction au surgissement d'émotions susceptibles de troubler les apprentissages ${ }^{45}$. Il peut s'agir d'hétéro-régulation ou d'auto-régulation des ressentis, à l'échelle de l'individu ou du groupe, avec ou sans intervention extérieure (dans le cas présent, d'une personne en situation

${ }^{40} \mathrm{C}$. Pierrisnard, «L'entretien de co-explicitation au service de la recherche collaborative », art. cit.

${ }^{41}$ C. Polo, K. Lund, C. Plantin, \& G. P. Niccolai, "Group emotions: the social and cognitive functions of emotions in argumentation". IJCSCL 11 (2)/2016, p. 123-156.C. Polo, C. Plantin, K. Lund \& G. P. Niccolai, "Group emotions in collective reasoning: a model", Aroumentation, 31(2)/2017, p. 301-320.

42 «Face » s'entend ici au sens de la politesse linguistique comme la valeur sociale positive attribuée aux personnes dans l'interaction.

${ }^{43}$ Polo, C. Polo, "Mise en scène argumentative des sujets et régulation émotionnelle en CRP", in A. Fournel \& J.-

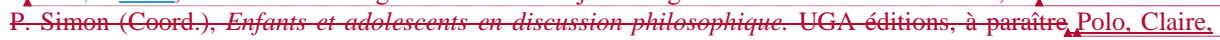
Lund, Kris, Plantin, Christian, Niccolai, Gerald P. (à paraître), Group Reasoning as the Alignment of Self-Identity Individual Footings. In P. Basso Fossali, C. Coupé, P. Griggs, K. Lund, A. Mazur-Palandre, V. Traverso (Coord.), Appeals to complexity, linguistic perspectives. Language Science Press.

${ }^{44} \mathrm{C}$. Polo et al., "Quand construire une position émotionnelle, c'est choisir une conclusion argumentative : le cas d'un café-débat sur l'eau potable au Mexique », Semen 35/2013, p. 41-63.

45 Voir plusieurs références à ce sujet : H. Järvenoja \& S. Jarvela, "Emotion control in collaborative learning situations: Do students regulate emotions evoked by social challenges?", British Journal of Educational Psychology, 79/2009, p. 463-481; H. Järvenoja \& S. Järvelä, "Regulating emotions together for motivated collaboration", In M. J. Baker, J. Andriessen, \& S. Järvelä (Eds.), Affective Learning Together, Routledge, 2013, p. 162-181; Gross, Handbook of emotion regulation, Guilford Press, 2007 ; C. A. Wolters, "Regulation of Motivation: Evaluating an Underemphasized Aspect of Self-Regulated Learning", Educational Psychologist, $38: 4 / 2003$, p. $189-205$.
Commenté [AAF1]: Je n'ai pas trouvé dans la biblio Polo et al, 2016 mais 2017

Commenté [AAF2]: Quelle est la référence? Polo et al. "Affective Dialoguing Together: Considering the Socio-Cognitivo-Emotional Nature of Collaborative Learning in the Analysis of Educational Interactions." Qui est en ligne?

Ou Polo, à paraître?

Mis en forme : Français (France)

Mis en forme : Anglais (États-Unis)

Mis en forme : Couleur de police : Automatique,

Anglais (États-Unis)

Mis en forme : Anglais (États-Unis)

Mis en forme : Couleur de police : Automatique, Anglais (États-Unis)

Mis en forme : Police :Italique, Couleur de police: Automatique, Anglais (États-Unis)

Mis en forme : Couleur de police : Automatique, Anglais (États-Unis)

Mis en forme : Couleur de police : Automatique 
d'animation). Le spectre de la régulation émotionnelle va de l'identification des émotions (prise de conscience de son propre état) à leur modification (en intensité ou en nature), en passant par leur explicitation, partage, planification, contrôle et évaluation à des fins de régulation de l'apprentissage.

Cependant, l'élargissement de la notion, en incluant un volet «préventif », permettrait de considérer le travail de régulation a priori relevant de la création et l'entretien d'un climat émotionnel propice au raisonnement. Au plan théorique, cela serait cohérent avec la reconnaissance, à bien des égards, d'un rôle positif des émotions dans l'activité cognitive, plutôt que de ne les appréhender que comme obstacles potentiels à son déroulement. Dans une certaine mesure, la conception et l'animation d'une situation « kairogène » dans l'interaction, pourrait relever d'une telle régulation émotionnelle préventive : plutôt que de porter sur la réaction à des affects susceptibles de faire obstacle au raisonnement, le travail d'instauration d'un tel climat cherche à favoriser les émotions favorables à l'enquête philosophique. Dans un tel cadre, il devient possible d'articuler la régulation des ressentis des sujets dialoguant avec les actions construisant ou transformant les tonalités émotionnelles des objets du dialogue. Ainsi, il serait possible de rendre compte sous l'angle de leurs effets (dé)régulateurs des efforts faits, par exemple, pour «réchauffer» ou «refroidir» les controverses dans leur traitement pédagogique ${ }^{46}$, soit les rendre à la fois plus ou moins affectivement intenses et socialement vives.

Reste que ce type d'analyse n'a de sens que réalisé à partir des traces langagières des émotions en question, soit de leur manifestation en discours, telles que communicables à autrui. Si l'on reprend la terminologie de Caffi et Janney ${ }^{47}$, il s'agit alors de s'intéresser à l'émotif (émotion exprimée) plutôt qu'à l'émotionnel (émotion ressentie), même si, en pratique, la distinction est peu opérante. En effet, il faudrait déployer une méthodologie additionnelle coûteuse, chronophage et présentant toutefois des erreurs potentielles, pour chercher à vérifier l'adéquation entre l'émotif et l'émotionnel, alors même que 1) la distance entre les deux est a priori limitée par la charge cognitive supplémentaire qu'implique de feindre un état non ressenti et 2) ce qui est pertinent pour la dynamique interactionnelle est bien l'émotif, ce qui est rendu visible à autrui. Dès lors, une pluralité de marques linguistiques peut permettre d'appréhender l'émotif : lexique, inférences émotionnelles à base verbale, inférences émotionnelles à base gestuelle, débit de parole et rapidité des enchaînements, prosodie, intonation, rires et production d'onomatopées ${ }^{48}$. Cependant, cela laisse entier le problème, classique en psychologie, de la causalité entre évènements et émotions, le fameux débat James-Lange vs Cannon ${ }^{49}$. La représentation commune d'un pic émotionnel consiste généralement à identifier un évènement déclencheur qui provoquerait une réaction émotionnelle donnée. Une telle conception repose notamment sur une perception de l'évènement comme antérieur à l'émotion. Mais les choses

${ }^{46}$ L. Simonneaux, «L'enseignement des questions socialement vives et l'éducation au développement durable », Pour, 2008/3, p. 179-185.

$47 \mathrm{Cl}$. Caffi \& R. W. Janney, “Toward a pragmatics of emotive communication”, Journal of pragmatics, No.21/1994, p. 325-373.

${ }^{48}$ K. Lund, M. Quignard \& D. W. Shaffer, "Gaining insight by transforming between temporal representations of human interaction”, Journal of Learning Analytics 4(3)/2017, p. 102-122.

49 C. Plantin, "Emotion and Affect", in Tracy, Karen, Ilie, Cornelia \& Sandel, Todd (eds.). The International Mis en forme : Normal, Justifié Encyclopedia of Language and Social Interaction, John Wiley \& Sons, 2015. 
sont plus complexes, car pour qu'un évènement donné externe aboutisse à une émotion particulière, un travail évaluatif interne, subjectif, a lieu. Ainsi, sous la pluie, tout le monde est mouillé, mais certaines personnes vont se réjouir de ce temps, alors que d'autres s'en trouveront attristées. De plus, la manifestation physiologique associée à la communication de l'émotion en est également constitutive : demandez à des gens de rire, ils finiront par éprouver quelque chose de l'ordre de la joie. L'approche de Scherer ${ }^{50}$ rend bien compte de ces différentes facettes des émotions, parmi lesquelles il distingue les composants cognitifs, physiologiques, motivationnels, expressifs, et ressentis, associés à des sous-systèmes spécifiques qui conditionneraient conjointement le vécu émotionnel ${ }^{51}$.

\subsection{Philosopher en dialoguant : une activité socio-cognitivo-émotionnelle}

Tout apprentissage dialogique peut être appréhendé comme revêtant une triple nature sociocognitivo-émotionnelle ${ }^{52}$. Plus spécifiquement, le dialogue philosophique, dans sa conception même, admet un rôle fondamental des émotions, qu'il s'agisse de travailler des dispositions attentionnelles particulières, supposant un certain état émotionnel, ou, grâce à une telle attitude, de se laisser toucher de façon indissociablement cognitive et émotive par un mouvement de pensée surprenant. Lipman ${ }^{53}$ utilise le terme d' «états mentaux », aussi bien cognitifs (doute) qu'affectifs (confiance, respect) pour désigner ces éléments essentiels à la pratique de la philosophie. En dialogue, aussi bien produire du kairos que le percevoir et y réagir, supposent, en effet, curiosité, créativité, capacité d'étonnement, et attitude collaborative. De plus, il est intéressant de pointer que la surprise, qui caractérise la perception du kairos dans son approche restrictive, a un statut à part parmi les émotions de base, de l'ordre du substrat affectif fondamental indéfini, qui devient, sous l'effet d'une évaluation subjective, une émotion particulière ${ }^{54}$. Cependant, si l'on en croit la théorie de l'esprit ${ }^{55}$ qui fonde les pratiques de $\mathrm{P} 4 \mathrm{C}$, elle ne saurait suffire, à elle seule, à faire avancer l'activité philosophique. En effet, un étonnement profond, capable de remettre en cause la pensée préexistante, serait indispensable pour susciter le doute intellectuel moteur de l'engagement dans l'enquête philosophique, et de son renouvellement. Ainsi, l'étonnement consisterait en une remise en question fondée sur un pic affectif d' « ébranlement », qui n'est ni pure stupéfaction comme la surprise, ni fascination face au merveilleux, mais suppose un engagement actif et intellectuel du sujet constatant avec trouble le bousculement de ses modes de pensée antérieurs ${ }^{56}$.

De plus, une étude montre que les façons dont la personne en position d'animation et les enfants réagissent à une contribution émotionnellement intense (mise en scène personnelle intime

${ }^{50}$ K. R. Scherer, "On the nature and function of emotion: A component process approach", Approaches to emotion, 2293(317)/184, p. 293-318.

51 Ibid.

${ }^{52}$ C. Polo \& K. Lund, "Affective Dialoguing Together: Considering the Socio-Cognitivo-Emotional Nature of Collaborative Learning in the Analysis of Educational Interactions", Symposium Analizing Educational Dialogues: Integrative and Critical Perspectives, conférence de l'EARLI, en ligne, 2021.

${ }^{53}$ M. Lipman, À l'école de la pensée. Enseigner une pensée holistique, De Boeck Supérieur, 2006.

${ }^{54} \mathrm{C}$. Plantin, "Emotion and Affect", art. cit.

$55 \mathrm{Si}$ le concept d'étonnement est aussi vieux que la philosophie, c'est chez Dewey qu'on en trouve une approche pragmatique, permettant de l'appréhender dans ses réalisations concrètes. Sa théorie de l'enquête fait en effet de l'étonnement la «pulsation essentielle» de toute activité d'exploration. Cf. J. Dewey, La quête de certitude. Une étude de la relation entre connaissance et action, trad. P. Savidan, Galimard, 2014, p. 37.

56 J. Thiévenaz, "L'étonnement", Le Télémaque, 49/2016, p. 17-30. 
évoquant des affects forts) influencent le devenir de l'idée qu'elle véhicule ${ }^{57}$. Ainsi, un léger pic émotionnel tend à rendre une proposition plus saillante et favoriser sa reprise, mais les contributions trop intenses se voient passées sous silence, ou donnent lieu à d'autres stratégies de refroidissement affectif. Finalement, la saillance proprement émotive des propositions nouvelles et de leur accueil comme telles par les autres participants et participantes au dialogue semble jouer un rôle de visibilisation important afin qu'elles puissent alimenter l'enquête collective. Le travail par autrui d'une idée clairement énoncée ou apparaissant encore à l'état de germe est en effet central dans toute activité de raisonnement à plusieurs passant par le dialogue.

Dans le dialogue philosophique, la régulation émotionnelle se pose ainsi à trois échelles temporelles. Sur le long terme, il s'agit de développer des compétences durables nécessaires à la pensée critique mais aussi à la créativité et à l'écoute cognitive profonde ${ }^{58}$. Sur une séance de philosophie, il s'agit de se mettre en état d'accueillir de nouvelles idées en soi et venant des autres, et de maintenir cet état dans le feu imprévu des échanges. Sur l'instant, face à la survenue du kairos, il s'agit de parvenir à le saisir non seulement comme surprise paralysante, mais comme évènement dont on s'étonne activement, méritant d'activer un doute renouvelant l'enquête. Dans une perspective didactique, on peut ajouter, pour la personne en situation d'animation, l'usage des émotions pour rendre plus saillants les éléments disruptifs de la discussion, et favoriser ainsi leur reprise et élaboration ultérieure. En quelque sorte, il ne suffirait pas ici de ressentir des émotions propices à saisir le kairos, mais de les communiquer de telle sorte que celui-ci soit perçu par l'ensemble du groupe. Dans une moindre mesure, les enfants eux-mêmes font bien sûr parfois également un tel travail de régulation émotionnelle consistant à donner à voir ce qu'une contribution a d'étonnant. Cependant, l'intention didactique caractérise sur ce point l'activité de la personne qui anime en tant que geste professionnel, par la conscience de devoir insister sur la pépite qui émerge afin de la rendre visible à l'ensemble des enfants.

\section{Cadre problématique et méthodologique : analyse affective et argumentative de gestes experts}

Le présent chapitre, focalisé sur la saisie émotive du kairos en $\mathrm{P} 4 \mathrm{C}$, porte plutôt sur une échelle de temps courte, correspondant à la réaction face à sa survenue. Cependant, dans une visée à la fois éducative et philosophique, nous nous penchons également sur les effets de cette réaction pour le devenir conversationnel du nouveau qui surgit alors, nous amenant à nous intéresser à tout le reste de la séance.

\subsection{Questions de recherche : émotions face au kairos et son devenir conversationnel}

Nous nous intéresserons aux affects associés au surgissement du kairos dans le dialogue philosophique avec des enfants, aux pratiques de régulation émotionnelle face à cet imprévu pourtant recherché, et à leurs effets conversationnels et pour le raisonnement collectif.

\footnotetext{
${ }^{57} \mathrm{C}$. Polo, « Mise en scène argumentative des sujets et régulation émotionnelle en CRP », art. cit.

${ }^{58}$ M. Lipman, Thinking in Education (2e éd.), Cambridge University Press, 2003.
} 
L'animation joue ici un rôle spécifique, mais nous n'excluons pas non plus le travail discursif et interactionnel des élèves sur ces questions. Plus spécifiquement, notre étude se décline en 4 questions de recherche :

QR1 - Qu'est-ce qui caractérise le kairos, sur le plan des réactions émotives, aussi bien des élèves que de la personne qui anime la séance, témoignant, dans l'interaction, la survenue d'un élément disruptif?

QR2 - A quelle régulation émotionnelle donne-t-il lieu par les élèves ?

QR3 - Quelle régulation émotionnelle en est réalisée dans le travail d'animation?

QR4 - Quels sont les effets conversationnels et sur le développement du raisonnement collectif de ces différentes saisies émotives du kairos?

\subsection{Un corpus de pratiques d'animation expertes}

Ce travail a été réalisé grâce à la mise à disposition d'un corpus d'enregistrement audiovisuel de 8 séances de $\mathrm{P} 4 \mathrm{C}$ par l'équipe Phileduc du laboratoire grenoblois LIDILEM, à l'occasion de colloques organisés en 2015, 2017 et 2019. Il rassemble des situations variées de dialogue philosophique avec des enfants, à l'école primaire et au collège, en classe ou en club philo, à partir de questions, d'œuvres littéraires, cinématographiques, de citations... Certaines relèvent de la «méthode Tozzi », tandis que d'autres sont plutôt d'inspiration lipmanienne. Deux séances sont des démonstrations observées par un public s'y intéressant du point de vue de la pratique d'animation en $\mathrm{P} 4 \mathrm{C}$ ou dans une perspective de recherche en sciences humaines et sociales, et concernent un groupe d'élèves relativement restreint (de l'ordre de la dizaine). Nous avons travaillé sur l'ensemble de ce corpus, et mis en lumière, en le visualisant, des schèmes d'action récurrents concernant la saisie émotive du kairos.

Cependant, nous focalisons notre propos ici sur ces deux séances de démonstration, et en particulier sur l'analyse d'extraits typiques et courts illustrant de façon emblématique la typologie des saisies émotives du kairos ainsi mise au point, et donnant à voir son opérationnalité sur des données authentiques. Étudier des pratiques reconnues comme expertes dans le champ permet également de s'assurer que les réactions émotives et la régulation émotionnelle décrites ne s'expliquent pas par un manque d'expérience ou de formation. Une séance est animée par Michel Tozzi, qui a joué un rôle fondamental dans la reconnaissance et la structuration de la $\mathrm{P} 4 \mathrm{C}$ en France, et porte sur la justice, à partir de la question « Pourquoi on dit 'c'est pas juste'? », puis relancée avec le problème du partage d'un gâteau. L'autre séance est animée par Michel Sasseville, qui s'inscrit, au Québec, en continuité de la tradition lipmanienne de la $\mathrm{P} 4 \mathrm{C}$, et part d'une question enfantine soulevée lors d'un travail préalable en club philo: «D'où viennent nos pensées? ». Avec ces deux démonstrations, ce sont donc également des écoles de P4C différentes qui s'illustrent ici. Or dans chacune de ces deux séances, nous avons trouvé des exemples des 6 types de saisies émotives du kairos répertoriées, qui ne sont donc a priori pas dépendantes de ces orientations pédagogiques différentes.

\subsection{Méthodologie : analyse interactionnelle et multimodale}

La définition même du kairos (cf. 1.1) rend impossible toute entreprise qui viserait à en faire l'inventaire dans un corpus donné : plus ou moins omniprésent ou rarissime selon que l'on en a une conception extensive ou restrictive, relevant d'une appréciation subjective délicate, dont tout le monde ne fait pas toujours preuve en situation, il se prête mal à un décompte. Bien 
souvent, l'apanage de la position de recherche est de repérer les kairos manqués, a posteriori : ces fils dont on aimerait en savoir davantage, qui paraissent soulever des idées enfantines prometteuses, mais que l'animation laisse de côté, voire ignore complètement. En effet, ces « ratés » évidents sont plus aisés à démasquer dans une telle posture que dans le feu de l'animation, en temps réel, qui, de plus, tente de tenir ensemble une diversité d'objectifs, et doit souvent les prioriser pour réagir dans l'instant.

Nous avons donc choisi de privilégier un point de vue émique, c'est-à-dire d'étudier la saisie émotive du kairos du point de vue des personnes qui interagissent, ce qui est au fondement de l'épistémologie de la linguistique interactionnelle ${ }^{59}$. Nous avons ici retenu la conception restrictive du kairos, puisque nous l'avons appréhendé en donnant de l'importance à son caractère surprenant. Ainsi, nous repérons les moments qui sont traités comme relevant du kairos, qu'ils soient plus ou moins exploités ensuite pour le raisonnement collectif, soit des moments :

- où s'exprime la surprise face à une contribution inattendue, éventuellement élaborée ensuite en une autre émotion mieux définie ;

- et/ou lorsque l'animation rebondit sur une contribution pour en souligner la nouveauté, la valoriser, demander de l'approfondir, la questionner, ou la situer par rapport à une éventuelle antithèse antérieure ${ }^{60}$;

- et/ou lorsque de telles réactions sont le fait des enfants ;

- et/ou lorsque les personnes qui ont formulé une nouveauté insistent pour qu'elle soit discutée car sa valeur pour l'enquête ne semble pas comprise par les autres ;

- et/ou lorsque les personnes qui ont formulé une nouveauté montrent un découragement faute de reprise et élaboration de leur apport.

Une telle approche ne saurait être que multimodale, notamment car les ressentis s'expriment souvent par des canaux paraverbaux plus implicites que les mots employés (intonation, expressions, onomatopées, posture des corps, etc.).

A partir de l'étude interactionnelle et multimodale approfondie de ce qui se passe dans de telles circonstances, sur l'ensemble des 8 séances à notre disposition, nous avons empiriquement défini des catégories d'analyse correspondant à des grands types de saisie émotive du kairos. Nous présentons cette typologie dans la partie suivante, en illustrant chacune des 6 configurations à partir d'extraits tirés des 2 séances de démonstration et analysés en détail.

\section{Résultats : typologie illustrée des saisies émotives du kairos en P4C}

\subsection{Présentation de la typologie}

Notre recherche a donné lieu à la mise au jour de 6 types de saisie émotive du kairos dans l'animation de dialogues philosophiques avec des enfants. Deux d'entre eux (numérotés 4 et 6), peuvent également être réalisées par des enfants, qu'il s'agisse de la personne qui a émis l'idée ou l'embryon d'idée nouvelle, ou d'autres enfants, immédiatement après son apparition, ou plus

${ }^{59}$ N. Markee, "Emic and Etic in Qualitative Research", in Carol A. Chapelle (Ed.), The Encyclopedia of Applied Linguistics, Blackwell Publishing Ltd, 2013.

${ }^{60} \mathrm{M}$. Tozzi, « Le kairos dans une DVDP : une éthique de l'intervention pour l'émergence de sujets pensants », art. cit. 
tard dans la discussion. Dans un ordre croissant d'élaboration ultérieure, les 6 types de saisies émotives du kairos suivants ont été identifiés :

1. Silence ;

2. Ratification simple ;

3. Refroidissement scolaire ;

4. Soulignage de la disruption ;

5. Réduction aux catégories préexistantes ;

6. Exploitation pour le raisonnement collectif.

La première consiste purement et simplement à ignorer l'idée disruptive malgré la manifestation d'une surprise à son endroit, éventuellement avec atténuation de l'offense ainsi réalisée. La saisie émotive de type 2 ratifie bien l'irruption de la nouveauté, avec une proto-évaluation sous forme d'appréciation affective, sans la reprendre. Une troisième possibilité réside dans le refroidissement scolaire du pic affectif associé à l'idée nouvelle qui émerge. Sa portée novatrice, potentiellement déstabilisante (mais aussi créatrice), se voit alors réduite par une transformation de l'activité en cours en travail scolaire avec des consignes spécifiques. Le refroidissement opère ici par mise à distance : on se désengage de la recherche philosophique proposée face à la mise à l'épreuve constituée par le kairos, et on se contente de faire un exercice (donner un exemple, une définition, etc.). Plutôt que de l'éviter, on peut se saisir du kairos en soulignant le caractère disruptif d'une contribution par l'expression d'une certaine émotivité (type 4). La surprise est alors élaborée en étonnement (attitude non partisane, mais qui favorise la mise à l'agenda de l'idée soulignée), en doute (questionnement ambivalent au plan argumentatif, pouvant aider à renforcer l'idée en émergence ou apparaître comme un acte d'opposition à cette idée), ou en appréciation positive de l'apport réalisé (l'idée est valorisée, voire soutenue). Une autre régulation possible de la surprise face au kairos consiste à le défaire de sa capacité à troubler les façons de penser antérieures en faisant rentrer de force la contribution disruptive dans des catégories de contenu préexistantes, quitte à la trahir. Cette cinquième configuration s'accompagne souvent de plusieurs reprises successives, fortement guidées par l'animateur ou l'animatrice, car un travail discursif important est nécessaire pour réaliser ce coup de force consistant à faire dire à la contribution sur laquelle on rebondit quelque chose de relativement différent de l'idée nouvelle, encore embryonnaire, qu'elle véhicule. Elle empêche la saisie du kairos, au sens philosophique du terme, et est même tout le contraire de sa définition comme acte philosophique. Cependant, elle alimente le raisonnement collectif, puisque la personne qui anime explicite à cette occasion des définitions ou distinctions philosophiques classiques, qui peuvent faire avancer la discussion (mais dans une autre direction). Enfin, le dernier type de saisie émotive du kairos consiste à la situer par rapport aux cheminements de pensée déjà ouverts et l'exploiter pour raisonner, en approfondissant l'enquête grâce à cette ouverture philosophique inédite (6).

Bien que décrits ici isolément, ces types de saisie émotive du kairos peuvent être combinés au fil des échanges, dans des dialogues authentiques. Par exemple, une première apparition d'une idée nouvelle peut être passée sous silence (1), mais, l'enfant insistant et reformulant sa pensée émergente de façon à souligner son caractère disruptif (4), elle pourra ensuite donner lieu à une exploitation pour le raisonnement collectif (6). En animation, on peut dans un premier temps être si dérouté(e) par une intervention qu'on se raccroche à un exercice habituel que l'on sait propice à la pensée philosophique (3), puis en profiter pour amener les enfants à reconstruire 
une distinction classique (5), à la manière d'un cours dialogué. Cependant, rien n'interdit que l'un ou l'une des enfants ait perçu l'intérêt de cette idée disruptive, et s'y réfère un peu plus tard, dans une formulation bien mûrie, qui l'exploite déjà pour l'enquête philosophique (6). Dans notre corpus, nous avons remarqué que des couples fréquents : refroidissement scolaire et réduction aux catégories existantes (3 et 5) d'une part; et surlignage de la disruption et exploitation pour le raisonnement collectif (4 et 6), d'autre part, vont souvent ensemble. Ce dernier couple tend à être également réalisé par les enfants, car c'est lui qui correspond véritablement à l'acte philosophique, dans une perspective adidactique. Le contrat de communication ne veut généralement pas que les enfants aient pour mission de ratifier les contributions des autres (ce qui incombe à l'animation), il est donc délicat ici de parler de type 1 (silenciation) en ce qui les concerne. De même, les enfants n'ont pas le pouvoir de changer la nature de l'activité, encore moins pour proposer des consignes scolaires, c'est pourquoi le type 3 (refroidissement scolaire) ne leur est pas accessible. Enfin, n'ayant pas de formation disciplinaire en philosophie, et ayant eu a priori peu l'occasion de réfléchir ou discuter préalablement à propos des problèmes qui leur sont livrés, ce sont des philosophes « profanes », dénués de catégories préexistantes (donc ne pouvant pas réaliser des saisies émotives de type $5)$.

\subsection{Silence ou ratification simple (types 1 et 2)}

La démonstration animée par Michel Tozzi offre un bon exemple de silenciation (type 1), mis en évidence et étudié en détail par Delille et ses collègues ${ }^{61}$, qui l'interprètent comme un kairos manqué. Alors que la discussion porte sur la juste façon de partager un gâteau, et que la question de l'inégalité entre des parts plus ou moins fournies en nappage ou bonbons décoratifs est abordée, un élève dit :

bah:: parce que:: (en)fin // le chocolat et tout ça bah on peut pas en mettre plus d'un côté ou pas (en)fin on essaie d'en mettre un peu // partout et bah après *chpense que c'est bien coupé normalement donc même si y a un endroit où y en a un peu plus c'est pas grave vu que à la base c'est un gâteau et c'est pas obligé (en)fin y a des gâteaux où y a rien d(e)ssus $\mathrm{i}(\mathrm{ls})$ sont natures donc ça sert à rien de vouloir une part où y a plein $\mathrm{d}(\mathrm{e})$ choses

Cette contribution n'invite à rien de moins qu'à la distinction philosophique entre la substance et l'accident, en questionnant ce qu'est en définitive une part de gâteau ${ }^{62}$. Cependant, son potentiel philosophique n'est pas relevé par l'animateur qui enchaîne après une courte pause que la qualité de l'enregistrement ne permet pas de mesurer précisément, en reposant à l'élève la question basique qu'il traite depuis plusieurs tours :

et tu penses que si on faisait des parts qui étaient inégales ça ça serait injuste ?

$\mathrm{Au}$ rang des ratifications simples, voyons un autre exemple, tiré cette fois de la démonstration animée par Michel Sasseville :

${ }^{61}$ V. Delille et al., «La pensée à l'œuvre dans une discussion philosophique : l'écouter, l'entendre, l'exploiter. Former à l'animation par l'observation minutieuse d'une discussion philosophique filmée », art. cit.

${ }^{62}$ Ibid. 
ULY : bah on peut / il faut plutôt maîtriser quand on pense mais aussi laisser aller ses pensées // ça fait mal à personne

ANI : ah \{rit $\}$ ça fait mal à personne // Nousseiba

Ulysse contre-argumente ici l'idée selon laquelle toute pensée véritable est contrôlée, et ouvre un nouveau cheminement de pensée en proposant d'explorer ce que pourrait être une pensée non maîtrisée. Un tel fil pourrait mener à considérer les approches contemplatives de la cognition, ou le rôle du lâcher prise dans la créativité, voire à considérer le fait de « laisser aller ses pensées » comme une pratique de bien-être psychique, antidote à un burn-out de surcharge mentale, qui lui pourrait «faire mal »... Bref, beaucoup d'éléments pourraient être exploités ici, mais l'animateur, s'il montre sa surprise par l'interjection « ah » produite en tournant la tête vers Ulysse, son rire, et l'intonation montante qui accompagne la reprise de la fin du tour de l'élève, passe directement à autre chose. Il a bien entendu, mais il ne fait rien de cet élément surprenant, et passe la parole à une autre élève (Nousseiba), vers qui il se tourne désormais.

\subsection{Refroidissement scolaire (3) combiné à la réduction aux catégories préexistantes (5)}

La combinaison de refroidissement scolaire (3) de l'idée disruptive associée à sa réduction aux catégories préexistantes de l'animateur (5) est illustrée par un extrait tiré de la démonstration de dialogue philosophique sur la justice. Il commence au tour 6 de la séance, et est transcrit cidessous :

62 ANI : ah est-ce que tu préfères que ce soit tiré au sort et que ce soit finalement un peu le hasard qui décide ou que ce soit une personne qui dise heu non c'est à toi et pas à toi ? 63 DIM : je préfère aucun des deux je préfère qu'on laisse la chose et qu'on la touche pas \{rires $\}$

64 ANI : d'accord et si tu devais choisir si tu devais (xxx) voilà soit c'est une personne qui décide dans ce cas-là soit on tire au sort est-ce qu'il y a quelque chose qui te semblerait moins juste ou ou moins juste que l'autre?

65 DIM : un p(e)tit peu plus juste le tirage au sort parce que comme ça si c'est pas la personne qui l'a:: qui a // dit c'est moi qui l'ai c'est c'est pas cette personne qui // l'a eu et bah moi je trouve que c'est juste // parce que la personne elle a voulu heu faire une i/ une i/ une inégalité

$66 \mathrm{ANI}$ : d'accord toi tu penses que donc l'injustice finalement elle vient beaucoup des actions des hommes mais que dès dès lors que c'est le hasard ou le tirage au sort c'est quand même moins injuste que quand c'est une personne qui le fait?

67 DIM : bah:: oui

L'animateur donne ici la parole à Dimitri en lui posant une question qui l'invite à se situer par rapport à une opposition qui imprègne la discussion jusque-là, à savoir si une injustice est plus ou moins acceptable selon qu'elle est liée au hasard ou à l'action humaine. Cependant, Dimitri entend s'affranchir de choisir entre la peste et le choléra, et exprime, au tour 62, un refus radical de toute inégalité, car il est injuste dans tous les cas que quelqu'un ait quelque chose que d'autres n'ont pas... Allant jusqu'à un nivellement par le bas, par la privation de tout le monde de la chose en question. Une telle prise de position présente un potentiel important pour renouveler l'enquête, mais constitue aussi une sorte de provocation géniale, heureuse, qui aide à sortir d'un dilemme qui comporte déjà une résignation face à l'injustice. La surprise est 
manifeste dans les rires que son propos provoque dans l'assemblée. Mais l'animateur refuse d'interroger la façon déployée jusque-là d'appréhender le problème, et lui redemande de choisir entre les deux alternatives du dilemme, au tour 64, tout en convenant qu'il s'agit alors d'un exercice de pensée, supposant de mettre de côté sa conviction ( «si tu devais choisir »).

Dimitri ne se laisse toutefois pas facilement enfermé dans cette alternative binaire, et, s'il joue le jeu de produire, formellement, une réponse à la question qui lui est réitérée, au tour 65 , il en profite pour insister sur son idée selon laquelle toute inégalité est injuste. Il y adjoint le principe de mérite : toute personne qui voudrait produire une inégalité mériterait finalement d'être ellemême frappée par l'inégalité, en manquant de ce que d'autres ont. C'est seulement dans ce cadre, si le sort frappait la personne coupable d'avoir voulu s'approprier ce qui n'était pas partageable, que le hasard serait « un petit peu plus juste » qu'une inégalité d'origine humaine. L'animateur ne relève toujours pas toute la richesse de cette intervention, et conclut, au tour 66, avec un coup de force, qui la réduit à un simple exemple supplémentaire de jugement de l'inégalité issue du hasard comme moins injuste que l'inégalité d'origine humaine. Dimitri finit par ratifier cette interprétation sans grand enthousiasme, avec l'allongement de la voyelle finale de « bah » (tour 67).

\subsection{Soulignage de la disruption (4) combiné à l'exploitation pour le raisonnement (6)}

Pour donner à voir le fonctionnement conjoint du soulignage de la disruption (4) associé à l'exploitation du kairos pour le raisonnement collectif (6), nous allons nous appuyer sur l'extrait de la démonstration sur la pensée retranscrit ci-dessous :

144 ARN : en fait on est obligé de penser // si non on (ne) peut rien faire // si:: si j'allais faire une bêtise // et que je me rappelle de quelque chose // je (ne) vais pas le faire 145 ANI : boh // donc il faut que tu penses

146 ARN : bah oui si::: *chais pas ma mère elle me dit quelque chose on a besoin de la pensée pour [ranger et trier] ce $(\mathrm{xx})$

147 ANI : ah // c'est vraiment intéressant parce que la question que j'ai posée c'était que // est-ce qu'on peut ne ne pas penser et toi tu dis \{Arnaud: on (ne) peut pas ne pas penser $\} / /$ ta question c'est ta question à la limite est plus ou moins intéressant Michel n'est c'est pas tellement on peut /on peut on peut on peut pas ne pas penser que si on (ne) pense pas ça a des conséquences

148 ARN : bah aussi heu

149 ANI : énormément // donc il faudrait toujours penser

150 ARN : toujours

151 ANI : toujours $(\mathrm{xx})$ pour toi c'est TRES important //est-ce qui /

Cet échange commence avec l'intervention surprenante d'Arnaud au tour 144, qui, au lieu de contribuer à la discussion en cours explorant si l'on peut ne pas penser, inverse la perspective, et affirme qu'on ne peut pas ne pas penser. L'animateur se tourne vers l'élève dans une attitude d'écoute perplexe (regard, tête et buste), puis ne cache pas sa surprise, dès le tour suivant, avec l'interjection «boh » prononcée avec une intonation montante. Il l'invite alors à poursuivre, préciser sa pensée, en lui proposant une suite logique possible à ce qu'il avance « donc il faut que tu penses ». Cette attitude encourage l'élève à poursuivre, même s'il lui est difficile d'énoncer encore très clairement sa pensée, comme en témoigne son hésitation avec 
l'allongement du «si » en début de tour, avant qu'il ne mobilise un exemple pour éclairer son propos (tour 146). Par une parenthèse métadiscursive, l'animateur, au tour suivant (147), spécifie sa surprise initiale en plusieurs émotions, opérant un soulignage émotif du caractère disruptif de l'intervention de l'élève. Au tout début de ce tour, tout en produisant l'interjection «ah » avec une intonation montante, il revient physiquement à lui en cessant de se pencher vers l'élève, en redressant le buste et en levant les yeux et la tête, comme s'il cherchait ses mots. Il se tourne ensuite à nouveau vers Arnaud en formulant d'abord une appréciation positive qui valorise la proposition d'Arnaud («c'est très intéressant »), puis il explicite le renversement de perspective effectué par cette contribution, s'étonnant ici à voix haute, de façon à partager son étonnement avec le groupe d'enfants (changement de question). Son propos est bimodal : il est largement soutenu et complété par des gestes référentiels, comme c'est généralement le cas tout au long de la séance, et notamment aux tours 149 et 151.

Au même tour (147), l'animateur commence déjà à exploiter la contribution d'Arnaud pour le raisonnement collectif, en effectuant une montée en généralité à partir de l'exemple fourni : on passe du risque de «faire une bêtise » (tour 144) aux «conséquences » possibles de ne pas penser, qui pourraient même être énormes (tour 149). Il s'engouffre ici dans la «brèche » offerte par le kairos pour ouvrir un nouveau volet de l'enquête : faudrait-il toujours penser? (tour 149). Arnaud ratifie pleinement cette interprétation de son propos au tour 150, reprenant le terme «toujours » comme une façon de réaffirmer sa position vis-à-vis de cette nouvelle question qu'il a participé à faire émerger dans la discussion. L'animateur, au tour suivant, ratifie sa position, tout en la relativisant, avec l'expression "pour toi », même s'il explicite avoir compris l'importance que cela revêt pour l'élève (haussant la voix sur le «TRES»). En explicitant que ce n'est qu'un point de vue, il prépare la passation de la parole à quelqu'un d'autre, qui pourrait se positionner différemment sur cette question, qu'il relance au groupe en fin de tour. L'occasion a été saisie et est maintenant susceptible d'alimenter le reste de la discussion.

\section{Discussion : de l'opportunité des émotions dans la saisie du kairos en P4C}

Si la typologie des saisies émotives du kairos lors de l'animation de dialogue philosophique avec des enfants met en évidence des pratiques plus ou moins susceptibles, sur l'instant, de nourrir le raisonnement collectif, il ne nous appartient pas d'en dénoncer certaines comme néfastes en elles-mêmes. En effet, on les retrouve toutes chez des animateurs experts, s'il en est, en pleine démonstration de P4C. Il nous paraît plus fructueux de nous contenter de les décrire, du point de vue des opportunités qu'elles offrent pour la suite de la séance, voire de la formation à la pensée, à plus long terme (4.1). Un type de saisie émotive du kairos fait la part belle à l'expression des émotions, à savoir le soulignage émotif de la disruption, réalisable par qui anime la séance aussi bien que par qui y participe. Il nous paraît primordial de nous y arrêter un peu plus longuement, pour discuter de la place à accorder aux émotions en $\mathrm{P} 4 \mathrm{C}$, et d'une régulation émotionnelle susceptible de magnifier la surprise au service de l'avancement de la pensée (4.2). 


\subsection{Opportunités offertes par les différents types de saisie émotive du kairos décrits}

Afin de discuter nos résultats, et si le kairos est un moment ou un évènement opportun, il convient de revenir sur ce que signifie l'opportunité en dialogue éducatif, pour donner du sens à cette typologie des saisies émotives du kairos. Dans un travail sur les opportunités manquées dans la résolution de problèmes collaborative, Baker et Bielaczyk ${ }^{63}$ définissent en creux l'opportunité comme l'utilisation des ressources disponibles offrant l'occasion d'atteindre un ou plusieurs but(s) d'apprentissage. Parmi les ressources listées dans la situation qu'ils étudient, trois peuvent également s'appliquer à la $\mathrm{P} 4 \mathrm{C}$ : le partenaire (ici toutes les personnes qui participent à l'interaction ainsi que l'animateur ou l'animatrice), les dires et faires antérieurs de la séance, les supports didactiques. Une difficulté pour les pratiques qui nous intéressent provient de la multiplicité des buts recherchés via le dialogue philosophique, parfois entre les personnes participant à la même séance, qui, de ce fait, ne vont pas nécessairement identifier les mêmes ressources comme pertinentes. En situation d'animation, tout le monde ne privilégie pas toujours les mêmes objectifs, ne met pas le curseur au même endroit, dans la tension entre visée d'enquête et visée éducative. Enfin, une même personne peut hiérarchiser différemment les buts correspondant à ces deux pôles, selon le moment de la séance. Dans le tableau 1, en s'inspirant des 3 ressources mentionnées plus haut, et en les complétant, nous présentons les ressources utilisées pour chacun des types de saisie émotive répertorié, et les buts qu'elles peuvent permettre d'atteindre. D'ordres variés (linguistiques, institutionnelles, professionnelles, didactiques, disciplinaires, interactionnelles), ces ressources peuvent être inégalement disponibles entre les enfants, et entre le groupe des enfants et la personne qui anime.

Tableau 1. Opportunité des différents types de saisie émotive du kairos

\begin{tabular}{|c|c|c|}
\hline Type de saisie émotive & Ressources & Buts \\
\hline 1. Silence & Institutionnelle : gestion de la parole & \begin{tabular}{|l|} 
Garantir le rythme \\
Sélection des interventions
\end{tabular} \\
\hline 2. Ratification simple & $\begin{array}{l}\text { Linguistiques : réponses minimales, } \\
\text { répétitions, reformulations, gestes, } \\
\text { etc. }\end{array}$ & $\begin{array}{l}\text { Garantir le rythme } \\
\text { Sélection des interventions } \\
\text { Reconnaissance }\end{array}$ \\
\hline 3. Refroidissement scolaire & $\begin{array}{l}\text { Professionnelles : répertoire de tâches } \\
\text { et consignes } \\
\text { Didactiques : supports, contrat de } \\
\text { communication habituel }\end{array}$ & $\begin{array}{l}\text { Passage à un objet moins } \\
\text { intense, plus facilement } \\
\text { appropriable par autrui } \\
\text { Travail d'exercices scolaires }\end{array}$ \\
\hline $\begin{array}{l}\text { 4. Soulignage émotif de la } \\
\text { disruption }\end{array}$ & $\begin{array}{l}\text { Linguistiques et culturelles : mots, } \\
\text { prosodie, expressions faciales, } \\
\text { position du corps, gestes, etc. }\end{array}$ & Rendre visible l'idée nouvelle \\
\hline $\begin{array}{l}\text { 5. Réduction aux catégories } \\
\text { préexistantes }\end{array}$ & $\begin{array}{l}\text { Expérientielles (âge) } \\
\text { disciplinaires : formation } \\
\text { philosophique }\end{array}$ & $\begin{array}{l}\text { Passage à un objet moins } \\
\text { intense, plus facilement } \\
\text { appropriable par autrui }\end{array}$ \\
\hline
\end{tabular}

${ }^{63}$ M. J. Baker \& K. Bielaczyc, "Missed opportunities for learning in collaborative problem-solving interactions.", in J. Greer (Ed.) Proceedings of AI-ED '95: World Conference on Artificial Intelligence in Education, AACE, 1995 , p. $210-217$. 


\begin{tabular}{|l|l|l|} 
& $\begin{array}{l}\text { linguistiques : codes polyphoniques } \\
\text { de mobilisation du discours autre }\end{array}$ & $\begin{array}{l}\text { Acquisition de concepts } \\
\text { Valorisation de l'enfant }\end{array}$ \\
\hline $\begin{array}{l}\text { 6. Exploitation pour le } \\
\text { raisonnement collectif }\end{array}$ & $\begin{array}{l}\text { Interactionnelles : partenaires, } \\
\text { dires et cheminements de pensée } \\
\text { antérieurs } \\
\text { Professionnelles : gestes de synthèse } \\
\text { et de « rebond » } \\
\text { Linguistiques : communication des } \\
\text { émotions (cf. 4) }\end{array}$ & $\begin{array}{l}\text { Exploration philosophique du } \\
\text { nouveau cheminement de } \\
\text { pensée ouvert par le kairos }\end{array}$ \\
\hline
\end{tabular}

Le silence (1) exploite la ressource institutionnelle qui donne à la personne en situation d'animation le pouvoir de gérer la répartition de la parole, rendant possible de passer directement à autre chose, et/ou à l'intervention de quelqu'un d'autre. Cela peut lui permettre de garantir le rythme nécessaire au maintien de l'attention et la concentration des enfants, de sélectionner dans le flot des idées foisonnantes celles jugées les plus propices à l'avancement de la discussion, et/ou provenant de personnes différentes, afin de favoriser la participation du plus grand nombre, ou encore de laisser aux seuls enfants la responsabilité de reconnaître ce qui constitue ou non un kairos.

La ratification simple (2) relève de la compétence linguistique de locuteur ou locutrice. Elle rend possible de manifester une reconnaissance de la contribution réalisée en signifiant qu'elle a été entendue, sans pour autant s'y arrêter, donc en opérant une sélection des idées ou personnes similaire au type 1 . Elle peut aussi participer au maintien du rythme adéquat de la discussion. La reconnaissance permise par le type 2 n'est pas nulle pour les enfants, qui, en sentant leur parole accueillie, peuvent être encouragés ainsi à continuer à réfléchir et à partager leurs idées. La ratification peut passer par des réponses minimales, des répétitions, des reformulations, des gestes.

Le refroidissement scolaire (3) suppose des ressources professionnelles sous la forme de la maîtrise d'un répertoire de tâches (fournir un exemple, donner une définition, introduire du vocabulaire, etc.), de la préexistence d'un contrat didactique confiant à la personne qui anime le pouvoir de définir l'activité du groupe, et parfois des supports didactiques spécifiques (utilisation d'un tableau, référence à une lecture antérieure, etc.). En guidant la discussion vers un objet de discours moins intense émotionnellement, cela peut favoriser son appropriation par le groupe, ou éviter un effet de blocage si tout le monde se retrouve bouche bée face à une contribution trop surprenante. Pour la personne qui anime, cela lui évite de se retrouver paralysée par sa propre surprise, et lui assure une maîtrise du dialogue, qui, au moins, s'en trouve orienté vers le travail d'exercices scolaires classiques.

Le soulignage de la disruption (4) utilise les émotions comme ressources pour rendre visible la nouveauté de la pensée qui émerge : d'abord la surprise, puis éventuellement des affects plus déterminés (émerveillement, valorisation, étonnement, doute). Ce sont ici également des compétences linguistiques et culturelles qui s'expriment, grâce auxquelles ces émotions sont communiquées aux autres, voire amplifiées et théâtralisées. Des ressources sémiotiques variées peuvent ici être mobilisées, la manifestation émotive étant une performance multimodale (mots mais également intonation, expressions faciales, position du corps, gestes, etc.).

La réduction aux catégories préexistantes (5) peut se fonder sur des discussions antérieures (l'âge étant ici une ressource), et/ou sur une formation disciplinaire en philosophie. Elle exploite 
également le rôle institutionnel qui veut que l'enseignant ait en définitive la bonne réponse, institutionnalisant comme savoir valable les propositions d'élèves qui y aboutissent. Enfin, au plan linguistique, cela suppose la maitrise des formes de représentation du discours autre dans son propre discours ${ }^{64}$, soit un subtil usage de la polyphonie. La régulation émotionnelle à l'œuvre ici consiste à déplacer l'affect de l'objet surprenant aux sujets de la discussion, qui sont conviés à se rallier à la pensée de la personne qui anime. En faisant dire à l'autre ce que l'on a en tête, en posture d'animation, on le ou la valorise, en s'appuyant sur son intervention, mais pour alimenter son propre raisonnement d'adulte. Cette forme de saisie émotive du kairos tend ainsi à associer les personnes à leurs idées, réelles ou attribuées. Cette dynamique est contraire à ce que prônent Dellile et ses collègues ${ }^{65}$ quant à la liberté philosophique d'imaginer des idées et de les explorer sans nécessairement les prendre en charge. La réduction aux catégories préexistantes refroidit également la discussion, en mettant un voile sur les éventuelles divergences de pensée, puisque tout est ramené au cadre d'analyse de la personne qui anime, donc à un objet moins intense émotionnellement. Si l'enfant qui s'est exprimé refuse de ratifier cette interprétation de ses propos, revient à la charge, ou si d'autres enfants en proposent une interprétation alternative, la scène prend des allures de « rébellion », souvent associée à des manifestations émotives (interjections, haussement de ton, rires, etc.).

Enfin, l'exploitation pour le raisonnement collectif (6) s'appuie sur les partenaires, les dires et les cheminements de pensée antérieurs, les gestes professionnels de synthèse et de « rebond » lorsqu'elle est le fait de l'animateur ou l'animatrice, et les compétences de communication émotive, au même titre que le type 4. En effet, rares sont les occurrences de type 6 qui ne sont pas associées à une forme, même minimale, de soulignage de la disruption. Ainsi, situer la potentialité du kairos par rapport à l'état de la discussion s'accompagne généralement d'un enthousiasme qui motive les enfants à s'engager dans la nouvelle voie ouverte pour l'exploration collective.

On peut s'interroger sur les opportunités ainsi décrites qu'offrent les différents types de saisie émotive du kairos du point de vue de leurs effets pour la recherche philosophique en cours et la formation à long terme. L'expérience des enfants au cours de la séance sera très différente selon qu'ils ont l'occasion de s'engager dans une enquête philosophique authentique ou qu'ils se retrouvent dans une nouvelle forme d'exercice scolaire finalement relativement classique (ex : essayer de deviner le mot qui est dans la tête de l'animateur ou l'animatrice). De la même façon, s'ils ont le sentiment que leur propos ne sont pas pris en compte, par une réaction silencieuse (1), par la réorientation de l'activité vers un exercice scolaire (3), par leur mise en doute comme étant problématique (4 soulignage de la disruption faisant évoluer la surprise en suspicion) ou qu'ils n'ont de valeur que lorsqu'ils semblent deviner ce que l'adulte a en tête (5), cela peut à long terme affecter leur estime d'eux-mêmes et leur participation orale. A l'inverse, lorsque l'adulte prend acte, même sans la traiter, de leur proposition (2), souligne son intérêt par des émotions encourageantes (4), et l'exploite pour relancer l'enquête collective (6), cela peut motiver les enfants à continuer à apprendre à philosopher par le dialogue et leur donner

${ }^{64} \mathrm{P}$. Roiné, « Reprises et représentations du discours de l'élève dans le discours de l'enseignant : un "triple jeu discursif" pendant les Discussions à Visée Philosophique », art. cit.

${ }^{65}$ V. Delille et al., «La pensée à l'œuvre dans une discussion philosophique : l'écouter, l'entendre, l'exploiter.

Former à l'animation par l'observation minutieuse d'une discussion philosophique filmée », art. cit. 
confiance dans leur capacité à penser par eux-mêmes, tout en se laissant toucher par les apports des autres.

4.2 Focus sur le soulignage émotif de la disruption (4) : comment magnifier la surprise ? En s'intéressant au kairos dans une conception restrictive, on pointe ces moments où

l'animateur $[\ldots]$ est, $[\ldots]$ au moins dans un premier temps, désarçonné, perdu, n'ayant pas encore identifié le problème philosophique auquel il est confronté ; ces moments où il est donc tenté de fuir ou d'oblitérer cette situation d'inconfort et de confusion ${ }^{66}$.

Dès lors, les saisies émotives de type 1, 2, 3 et 5 (silence, ratification simple, refroidissement scolaire, réduction aux catégories préexistantes) peuvent être comprises comme des moyens de s'en sortir malgré une telle surprise déstabilisante. La régulation émotionnelle consiste alors à chercher à diminuer ce pic affectif, voire à le faire disparaître, afin de maîtriser la situation et l'évolution de la discussion... quitte à passer à côté d'une pensée inédite riche. Si les enfants insistent pour tenter de la remettre sur la table, on tendra à déclarer leurs propos peu clairs, ou, au moins, à signifier une incompréhension, sans doute sincère, qui les invite à reformuler, mais peut aussi être décourageante.

Au plan de la régulation émotionnelle, le type 4 de saisie du kairos, soit le soulignage de la disruption par la manifestation émotive, a ceci de particulier qu'il ne suppose pas le contrôle immédiat de l'affect qui surgit par une raison supposée supérieure. Au contraire, la régulation est ici surtout l'explicitation de cet affect, en présumant sa valeur potentielle pour le raisonnement. En laissant s'exprimer, voire en amplifiant sa surprise, en s'émouvant dans le kairos, on le rend au moins visible, à défaut d'être immédiatement intelligible. Cela suppose une grande confiance dans les partenaires de la discussion pour leur avouer un tel trouble, en estimant le groupe capable, dans un second temps, de lui donner un sens. Réguler l'affect ne consiste alors plus à cacher sa surprise, mais à la magnifier collectivement, en la spécifiant ensuite, dans l'échange, en étonnement philosophique, porteur évidemment de doute, mais sous une forme plus féconde qu'un réflexe de scepticisme dévalorisant une pensée nouvelle avant tout examen. Une telle attitude valorise l'émergence de la piste inconnue qui s'ouvre comme une grâce, et s'émerveille du trouble qu'elle cause en soi le plus explicitement possible, afin à la fois de l'accueillir avec gratitude, et de la partager avec générosité. Le trouble partagé dont on s'émerveille ensemble peut alors devenir objet de discours, activement pensé comme étonnant, relançant l'enquête. Delille et ses collègues ${ }^{67}$ appellent de leurs vœux une telle « esthétique collective de l'étonnement ». Lorsque la personne en situation d'animation réagit ainsi, elle réalise à la fois un geste didactique et un acte philosophique, à la croisée des visées d'enquête et d'éducation de tels dialogues. Or, en tant qu'acte philosophique, le soulignage émotif de la disruption offre en lui-même une portée éducative pour l'exemple qu'il donne, car on apprend aussi par imitation, imprégnation. En acceptant le bousculement émotionnel associé à la remise en cause cognitive, l'adulte montre aux enfants qu'il n'y a pas lieu de fuir devant le kairos, et qu'il est possible de se laisser guider affectivement pour explorer des pensées prometteuses en construction. Cette attitude peut permettre aux enfants de développer ensuite une telle saisie émotive du kairos comme disposition durable propice à la pensée autonome.

${ }^{66}$ Ibid., p. 241-242

${ }^{67} \mathrm{Ibid}$. 


\section{Conclusion}

Le kairos, entendu dans une perspective restrictive, est caractérisé, au plan émotionnel, par la surprise, aussi bien pour les enfants que pour la personne qui anime le dialogue, en P4C. Plusieurs traces linguistiques étaient une telle réaction affective : pauses, intonations, rires, etc. Cette surprise peut donner lieu à différentes formes de régulation émotionnelle. La «saisie émotive du kairos » désigne l'ensemble composé par cette réaction affective initiale, ses manifestations émotives, ainsi que les émotions spécifiques auxquelles sa régulation émotionnelle donne lieu, telles que communiquées dans l'interaction. Certaines formes de régulation de sa propre surprise par l'animateur ou l'animatrice tendent plutôt à la limiter ou la maîtriser (types 1, 2,3,5), ce qui ne permet pas d'explorer le potentiel offert par ce kairos pour l'enquête philosophique en cours, mais présente des opportunités quant à d'autres objectifs assignés au dialogue (cf. tableau 1). D'autres formes de régulation émotionnelle, celles relevant du type 4, consistent à rendre explicite la surprise associée au kairos, puis à souligner l'opportunité cognitive qu'elle ouvre par une forme d'émerveillement, et enfin à l'élaborer en émotions plus définies utiles pour l'enquête : étonnement, doute. Ce type de saisie émotive du kairos est souvent suivi d'une véritable exploitation du kairos pour le raisonnement collectif (type 6), qui se trouve alors situé par rapport aux cheminements de pensée antérieurs et pleinement exploré. Enfin, il arrive que lorsque le kairos n'est pas immédiatement reconnu et exploité comme tel par l'adulte (types 1, 2, 3,5), sa reprise enfantine ultérieure, par la même personne ou autrui, donne finalement lieu à une exploitation pour le raisonnement collectif (type 6), parfois même amenant l'adulte à s'y engager pleinement.

Le rôle de l'expression des émotions dans la saisie du kairos avec des enfants peut nous amener à réfléchir sur la place qui leur est accordée dans le dialogue philosophique en général. Ainsi, le soulignage émotif de la disruption serait un acte philosophique donnant à voir certaines dispositions individuelles nécessaires pour penser, cultivées sur le long terme. Dans un vocabulaire lipmanien ${ }^{68}$, l'accueil de sa propre surprise et l'émerveillement devant elle relèvent des dispositions «attentives » et «créatives », et son élaboration en étonnement puis en doute des dispositions «critiques ». L'esthétique collective de l'étonnement nécessaire à la survenue et à la saisie du kairos dans le dialogue philosophique avec les enfants relève ainsi de la pensée multidimensionnelle, et nécessite le respect de sa nature socio-cognitivo-émotionnelle. Ni pensée attentive, ni pensée créative ni pensée critique ne sauraient se déployer avec la seule raison individuelle surplombante, et toutes émergent dans l'interaction indistinctement émotive et cognitive.

\section{Bibliographie}

${ }^{68}$ M. Lipman, Thinking in Education, op. cit. 
Baker, Michael J., «Les pédagogies dialogiques : raisonnement, conceptualisation et argumentation », in A. Fournel \& J.-P. Simon (Coord.), Enfants et adolescents en discussion philosophique, UGA éditions, à paraître.

Baker, Michael J. \& Bielaczyc, Katerine, "Missed opportunities for learning in collaborative problem-solving interactions.", in J. Greer (Ed.) Proceedings of AI-ED '95: World Conference on Artificial Intelligence in Education, AACE, 1995, p. 210-217.

Caffi, Claudia \& Janney, Richard W., "Toward a pragmatics of emotive communication", Journal of pragmatics, No.21/1994, p. 325-373.

Delille, Véronique, Markevitch Frieden, Nathalie, \& Jeanmart, Gaëlle, «La pensée à l'œuvre dans une discussion philosophique : l'écouter, l'entendre, l'exploiter. Former à l'animation par l'observation minutieuse d'une discussion philosophique filmée », in J.-P. Simon \& M. Tozzi (Coord.), Paroles de philosophes en herbe. Editions de l'Université de Grenoble, 2017, p. 239260.

Dewey, John, La quête de certitude. Une étude de la relation entre connaissance et action, trad. P. Savidan, Galimard, 2014 (première édition 1929).

Fournel, Anda \& Perret-Clermont, Anne-Nelly, «Quel est le problème du problème ? Une analyse du problème supposé commun dans un atelier philosophique avec des collégiens », in A. Fournel \& J.-P. Simon (Coord.), Enfants et adolescents en discussion philosophique, UGA éditions, à paraître.

Gross, Handbook of emotion regulation, Guilford Press, 2007.

Järvenoja, Hanna \& Järvelä, Sanna, "Regulating emotions together for motivated collaboration”, In M. J. Baker, J. Andriessen, \& S. Järvelä (Eds.), Affective Learning Together, Routledge, 2013, p. 162-181.

Järvenoja, Hanna \& Jarvela, Sanna, "Emotion control in collaborative learning situations: Do students regulate emotions evoked by social challenges?", British Journal of Educational Psychology, 79/2009, p. 463-481.

Jorro, Anne, Professionnaliser le métier d'enseignant, ESF, 2002.

Kebir, Yasmine, Boutet, Vincent, Specogna, Antonietta, Saint Dizier de Almeida, Valérie, « Le guidage en DVP : apport de l'analyse interlocutoire et des verbalisations en autoconfrontation », in A. Fournel \& J.-P. Simon (Coord.), Enfants et adolescents en discussion philosophique, UGA éditions, à paraître.

Kohler, Alaric, «Cheminement de pensée des élèves lors de leur schématisation du destin : une analyse de logique naturelle », in A. Fournel \& J.-P. Simon (Coord.), Enfants et adolescents en discussion philosophique, UGA éditions, à paraître.

Lebas-Fraczak, Lidia, «Les enfants font-ils spontanément des synthèses dans les discussions à visée philosophique ? », in A. Fournel \& J.-P. Simon (Coord.), Enfants et adolescents en discussion philosophique, UGA éditions, à paraître.

Lévine, Jacques, «La notion de «monde philosophique des enfants » : utopie ou nécessité ? Présupposés, place, limites ? », in M. Tozzi, Apprendre à philosopher par la discussion, De Boeck Supérieur, 2007, p. 93-107.

Lipman, Matthew, Thinking in Education (2e éd.), Cambridge University Press, 2003.

Lipman, Matthew, À l'école de la pensée. Enseigner une pensée holistique, De Boeck Supérieur, 2006. 
Lund, Kris, Quignard, Matthieu \& Shaffer, David W., "Gaining insight by transforming between temporal representations of human interaction", Journal of Learning Analytics 4(3)/2017, p. 102-122.

Markee, Numa, "Emic and Etic in Qualitative Research", in Carol A. Chapelle (Ed.), The Encyclopedia of Applied Linguistics, Blackwell Publishing Ltd, 2013.

Pana-Martin, Francine, Les gestes professionnels des formateurs d'enseignants en situation d'accompagnement individualisé, Thèse de sociologie, CNAM, 2015.

Pierrisnard, Christine, «L'entretien de co-explicitation au service de la recherche collaborative », Phronesis 6 (1)/2017, p. 153-165.

Plantin, Christian, "Emotion and Affect", in Tracy, Karen, Ilie, Cornelia \& Sandel, Todd (eds.). The International Encyclopedia of Language and Social Interaction, John Wiley \& Sons, 2015. Polo, Claire, «Mise en scène argumentative des sujets et régulation émotionnelle en CRP », in A. Fournel \& J.-P. Simon (Coord.), Enfants et adolescents en discussion philosophique, UGA éditions, à paraître.

Polo, Claire, «Les gestes métaphoriques comme construction collective d'analogies cognitives », in A. Fournel, J.-P. Simon, S. Lagrange-Lanaspre, J.-M. Colletta (Coord.), Philosopher avec les enfants, Presses universitaires Blaise-Pascal, 2020, p. 461-484.

Polo, Claire, "Gesture's Contribution to Collective Metaphorical Thinking in a Community of Philosophical Inquiry”, Studia UBB. Philosophia, 64 (3)/2019, p. 41-64.

Polo, Claire, «Jouer le jeu et enfreindre les règles : stratégies d'orientation discursive et argumentation dans une démonstration d'atelier philosophique », in J.-P. Simon \& M. Tozzi (Eds.), Paroles de philosophes en herbe, Editions de l'Université de Grenoble, 2017, p. 99-118. Polo, Claire \& Lagrange-Lanaspre, Sandra, "Metaphorical Reasoning Together: Embodied Conceptualization in a Community of Philosophical Inquiry", in K. Lund, G. Niccolai, E. Lavoué, C. Hmelo-Silver, G. Gweon, M. Baker (Eds), A Wide Lens: Combining Embodied, Enactive, Extended, and Embedded Learning in Collaborative Settings: 13th International Conference on CSCL, (1), 2019, p. 424-431.

Polo, Claire, Lund, Kris, Plantin, Christian, Niccolai, Gerald P. (à paraître), Group Reasoning as the Alignment of Self-Identity Individual Footings. In P. Basso Fossali, C. Coupé, P. Griggs, K. Lund, A. Mazur-Palandre, V. Traverso (Coord.), Appeals to complexity, linguistic perspectives. Language Science Press.

Polo, Claire \& Lund, Kris, "Affective Dialoguing Together: Considering the Socio-CognitivoEmotional Nature of Collaborative Learning in the Analysis of Educational Interactions", Symposium Analizing Educational Dialogues: Integrative and Critical Perspectives, conférence de l'EARLI, en ligne, 2021.

Polo, Claire, Plantin, Christian, Lund, Kris, \& Niccolai, Gerald P., "Group emotions in collective reasoning: a model”, Argumentation, 31(2)/2017, p. 301-329.

Polo, Claire, Lund, Kris, Plantin, Christian \& Niccolai, Gerald P., "Group emotions: the social and cognitive functions of emotions in argumentation". IJCSCL 11 (2)/2016, p. 123-156.

Polo, Claire, Plantin, Christian, Lund, Kris \& Niccolai, Gerald, P., «Quand construire une position émotionnelle, c'est choisir une conclusion argumentative : le cas d'un café-débat sur l'eau potable au Mexique », Semen 35/2013, p. 41-63.

Roiné, Philippe, «Reprises et représentations du discours de l'élève dans le discours de l'enseignant : un "triple jeu discursif" pendant les Discussions à Visée Philosophique », in A.

Commenté [CP3]: Note 41

Commenté [CP4]: Je l'ai ajouté conformément à la note 39 mais si on manque de place, on peut laisser uniquement la référence d'au-dessus de 2017 et la mettre en note 39 à la place

Mis en forme : Police :Italique

Mis en forme : Français (France)

Mis en forme : Français (France) 
Fournel \& J.-P. Simon (Coord.), Enfants et adolescents en discussion philosophique, UGA éditions, à paraître.

Scherer, Klaus R., "On the nature and function of emotion: A component process approach", Approaches to emotion, 2293(317)/184, p. 293-318.

Simonneaux, Laurence, «L'enseignement des questions socialement vives et l'éducation au développement durable », Pour, 2008/3, p. 179-185.

Thiévenaz, Joris, «L'étonnement », Le Télémaque, 49/2016, p. 17-30.

Tozzi, Michel, « Une approche par compétences en philosophie ? », Rue Descartes, 73(1)/2012, p. 22-51.

Tozzi, Michel, «Le kairos dans une DVDP : une éthique de l'intervention pour l'émergence de sujets pensants », Diotime (en ligne) 60/2014 (4).

Wolters, Christopher A., "Regulation of Motivation: Evaluating an Underemphasized Aspect of Self-Regulated Learning”, Educational Psychologist, 38:4/2003, p. 189-205. 